\title{
HOMOGENEOUS REINHARDT DOMAINS CONTAINING NO COORDINATE HYPERPLANES
}

\author{
SATORU ShimizU* and Kouichi Kimura
}

\begin{abstract}
As is well-known, a homogeneous Reinhardt domain in $\mathbf{C}^{*}$ coinsides with $\mathbf{C}^{*}$. In this paper, generalizing this fact, we show that a pseudoconvex homogeneous Reinhardt domain in $\left(\mathbf{C}^{*}\right)^{n}$ coinsides with $\left(\mathbf{C}^{*}\right)^{n}$ itself.
\end{abstract}

\section{Introduction}

In the study of Reinhardt domains $D$, investigating the structures of their holomorphic automorphism groups $\operatorname{Aut}(D)$ has fundamental importance. When $D$ is bounded, the structure of $\operatorname{Aut}(D)$ was clarified by, for example, Sunada [6], Shimizu [2], [3]. But, in the general case, or when $D$ is not necessarily bounded, a little is known about the structure of $\operatorname{Aut}(D)$. For instance, related to the investigation of the structure of $\operatorname{Aut}(D)$, we have the fundamental problem of determining a homgeneous Reinhardt domain, that is, the problem that when a Reinhardt domain $D$ admits a transitive action by $\operatorname{Aut}(D)$, what form does $D$ have? When $D$ is bounded, the following result is shown [3] (for the definition of the algebraic equivalence relation between Reinhardt domains, see Section 2 below):

Theorem 1.1. Let $D$ be a bounded Reinhardt domain in $\mathbf{C}^{n}$. If $D$ is homogeneous, then $D$ is algebraically equivalent to the direct product $B_{n_{1}} \times \cdots \times B_{n_{k}}$ of balls, where $B_{n_{i}}$ denotes the unit ball in $\mathbf{C}^{n_{i}}$.

On the other hand, as for the general case, there is a conjecture as follows:

Conjecture. For every homogeneous pseudoconvex Reinhardt domain $D$ in $\mathbf{C}^{n}$, there exist $k$ positive integers $n_{1}, \ldots, n_{k}(k$ may be 0$)$ and non-negative integers $l, m$ such that $n=n_{1}+\cdots+n_{k}+l+m$ and $D$ is algebraically equivalent

2000 Mathematics Subject Classification. Primary 32A07, 32M05; Secondary 32M10.

Key words and phrases. Reinhardt domains, automorphism groups.

* Partly supported by the Grant-in-Aid for Scientific Research (C), Japan Society for the Promotion of Science.

Received May 31, 2013; revised September 11, 2013. 
to the direct product $B_{n_{1}} \times \cdots \times B_{n_{k}} \times \mathbf{C}^{l} \times\left(\mathbf{C}^{*}\right)^{m}$, where $\mathbf{C}^{*}$ denotes the puctured complex plane.

Theorem 1.1 implies that this conjecture is true when $D$ is bounded. But it remains open in the unbounded case. The purpose of this paper is to give a partial answer in such case by applying the method given in Shimizu [5]. Namely, we prove the following:

Theorem 1.2. Let $D$ be a pseudoconvex Reinhardt domain in $\left(\mathbf{C}^{*}\right)^{n}$. If $D$ is homogeneous, then $D$ coincides with $\left(\mathbf{C}^{*}\right)^{n}$.

Note that the above theorem gives a higher-dimensional generalization of the classical fact that a Reinhardt domain $D$ in $\mathbf{C}^{*}$ is inhomogeneous except when $D$ coincides with $\mathbf{C}^{*}$ itself.

This paper is organized as follows. In Section 2, we recall basic concepts and results on Reinhardt domains. In particular, we collect some preliminary results used for proving Theorem 1.2 mainly from Shimizu [5]. Section 3 is devoted to the proof of Theorem 1.2.

\section{Preliminaries}

We first collect some notations and terminology. As a general notational convention, we denote elements of $\mathbf{Z}^{n}, \mathbf{R}^{n}$, or $\mathbf{C}^{n}$ by column vectors. When dealing with matrices, we denote by $I_{p}$ and $O$ the unit matrix of degree $p$ and the zero matrix, respectively. The set of non-zero complex numbers is denoted by $\mathbf{C}^{*}$. The multiplicative group of complex numbers of absolute value 1 is denoted by $U(1)$. An automorphism of a complex manifold $M$ means a biholomorphic mapping of $M$ onto itself. The group of all automorphisms of $M$ is denoted by $\operatorname{Aut}(M)$. Two complex manifolds are said to be holomorphically equivalent if there is a biholomorphic mapping between them.

We now recall basic concepts and results on Reinhardt domains (cf. Shimizu [2], [3]). Write $T=(U(1))^{n}$. The group $T$ acts as a group of automorphisms on $\mathbf{C}^{n}$ by the standard rule

$$
\alpha \cdot z={ }^{t}\left(\alpha_{1} z_{1}, \ldots, \alpha_{n} z_{n}\right) \text { for } \alpha={ }^{t}\left(\alpha_{1}, \ldots, \alpha_{n}\right) \in T \text { and } z={ }^{t}\left(z_{1}, \ldots, z_{n}\right) \in \mathbf{C}^{n} .
$$

By definition, a Reinhardt domain $D$ in $\mathbf{C}^{n}$ is a domain in $\mathbf{C}^{n}$ which is stable under the action of $T$, that is, such that $\alpha \cdot D \subset D$ for all $\alpha \in T$. The group $T$ then acts as a group of automorphisms on $D$. The subgroup of $\operatorname{Aut}(D)$ induced by the action of $T$ is denoted by $T(D)$.

An automorphism $\varphi$ of $\left(\mathbf{C}^{*}\right)^{n}$ is called an algebraic automorphism of $\left(\mathbf{C}^{*}\right)^{n}$ if the components of $\varphi$ are given by Laurent monomials, that is, $\varphi$ is of the form

$$
\begin{gathered}
\varphi:\left(\mathbf{C}^{*}\right)^{n} \ni{ }^{t}\left(z_{1}, \ldots, z_{n}\right) \mapsto{ }^{t}\left(w_{1}, \ldots, w_{n}\right) \in\left(\mathbf{C}^{*}\right)^{n}, \\
w_{i}=\alpha_{i} z_{1}^{a_{i 1}} \cdots z_{n}^{a_{i n}}, \quad i=1, \ldots, n,
\end{gathered}
$$


where $\left(a_{i j}\right) \in G L(n, \mathbf{Z})$ and $\left(\alpha_{i}\right) \in\left(\mathbf{C}^{*}\right)^{n}$. The set $\operatorname{Aut}_{\mathrm{alg}}\left(\left(\mathbf{C}^{*}\right)^{n}\right)$ of all algebraic automorphisms of $\left(\mathbf{C}^{*}\right)^{n}$ forms a subgroup of $\operatorname{Aut}\left(\left(\mathbf{C}^{*}\right)^{n}\right)$.

Let $\varphi$ be an algebraic automorphisms of $\left(\mathbf{C}^{*}\right)^{n}$ and write $\varphi(z)={ }^{t}\left(\varphi_{1}(z), \ldots\right.$, $\left.\varphi_{n}(z)\right)$. In general, the components $\varphi_{1}, \ldots, \varphi_{n}$ have zeroes or poles along each coordinate hyperplane. If, for two domains $D$ and $D^{\prime}$ in $\mathbf{C}^{n}$ not necessarily contained in $\left(\mathbf{C}^{*}\right)^{n}$, they have no poles on $D$ and $\varphi: D \rightarrow \mathbf{C}^{n}$ maps $D$ biholomorphically onto $D^{\prime}$, then we say that $\varphi$ induces a biholomorphic mapping of $D$ onto $D^{\prime}$.

Consider a biholomorphic mapping $\varphi: D \rightarrow D^{\prime}$ between two Reinhardt domains $D$ onto $D^{\prime}$ in $\mathbf{C}^{n}$. The following proposition gives a necessary and sufficient condition for $\varphi$ to be equivariant with respect to the $T$-actions.

Proposition 2.1 (cf. [3, Section 2]). $\varphi$ is induced by an algebraic automorphism of $\left(\mathbf{C}^{*}\right)^{n}$ if and only if it has the property that $\varphi T(D) \varphi^{-1}=T\left(D^{\prime}\right)$.

Biholomorphic mappings between Reinhardt domains equivariant with respect to the $T$-actions may be considered as natural isomorphisms in the category of Reinhardt domains. In view of this observation, we say that two Reinhardt domains in $\mathbf{C}^{n}$ are algebraically equivalent if there is a biholomorphic mapping between them induced by an algebraic automorphism of $\left(\mathbf{C}^{*}\right)^{n}$.

There is a useful correspondence between Reinhardt domains and tube domains. A tube domain $T_{\Omega}$ in $\mathbf{C}^{n}$ is a domain in $\mathbf{C}^{n}$ given by $T_{\Omega}=$ $\Omega+\sqrt{-1} \mathbf{R}^{n}$, where $\Omega$ is a domain in $\mathbf{R}^{n}$. We call $\Omega$ the base of $T_{\Omega}$. For each element $\eta$ of $\mathbf{R}^{n}$, we define an automorphism $\sigma_{\eta}$ of $T_{\Omega}$ given as a translation of $\mathbf{C}^{n}$ by $\sigma_{\eta}(\zeta)=\zeta+\sqrt{-1} \eta$ for $\zeta \in T_{\Omega}$. Now consider a mapping ord : $\left(\mathbf{C}^{*}\right)^{n} \rightarrow$ $\mathbf{R}^{n}$ defined by

$$
\operatorname{ord}\left({ }^{t}\left(z_{1}, \ldots, z_{n}\right)\right)=\left(-\frac{1}{2 \pi} \log \left|z_{1}\right|, \ldots,-\frac{1}{2 \pi} \log \left|z_{n}\right|\right) \text { for }{ }^{t}\left(z_{1}, \ldots, z_{n}\right) \in\left(\mathbf{C}^{*}\right)^{n} \text {. }
$$

If $D$ is a Reinhardt domain in $\left(\mathbf{C}^{*}\right)^{n}$, then $\operatorname{ord}(D)$ is a domain in $\mathbf{R}^{n}$, and it is well-known that $D$ is pseudoconvex if and only if $\operatorname{ord}(D)$ is a convex domain in $\mathbf{R}^{n}$. To each Reinhardt domain $D$ in $\left(\mathbf{C}^{*}\right)^{n}$, there is associated a tube domain $T_{\Omega}$ in $\mathbf{C}^{n}$ with $\Omega=\operatorname{ord}(D)$. The tube domain $T_{\Omega}$ naturally becomes a covering manifold of $D$. Indeed, introduce a covering $\varpi: \mathbf{C}^{n} \rightarrow\left(\mathbf{C}^{*}\right)^{n}$ defined by

$$
\varpi\left({ }^{t}\left(\zeta_{1}, \ldots, \zeta_{n}\right)\right)={ }^{t}\left(e^{-2 \pi \zeta_{1}}, \ldots, e^{-2 \pi \zeta_{n}}\right) \quad \text { for }{ }^{t}\left(\zeta_{1}, \ldots, \zeta_{n}\right) \in \mathbf{C}^{n}
$$

Then we have $T_{\Omega}=\varpi^{-1}(D)$, and the restriction $\varpi: T_{\Omega} \rightarrow D$ is a covering projection. The covering transformation group for $\varpi: T_{\Omega} \rightarrow D$ is given by $\left\{\sigma_{\eta} \mid \eta \in \mathbf{Z}^{n}\right\}$. We call $T_{\Omega}$ the covering tube domain of $D$ and $\varpi: T_{\Omega} \rightarrow D$ the canonical covering projection. Note that if $D$ is pseudoconvex, then $\varpi: T_{\Omega} \rightarrow D$ gives the universal covering of $D$. Indeed, if $D$ is pseudoconvex, then $T_{\Omega}$ is simply connected, because $\Omega$ is convex, and consequently simply connected.

Let $D$ be a Reinhardt domain in $\left(\mathbf{C}^{*}\right)^{n}$ and write $\Omega=\operatorname{ord}(D)$. Suppose that $D$ is pseudoconvex. It follows that, for the convex domain $\Omega$, there exists 
an affine transformation $f$ of $\mathbf{R}^{n}$ such that

$$
f(\Omega)=\Xi^{(1)} \times \mathbf{R}^{l},
$$

where $l$ is an integer between 0 and $n$ and $\Xi^{(1)}$ is a convex domain in $\mathbf{R}^{n-l}$ containing no complete straight lines (cf. [1]). This implies that if, for each point $\xi$ of $\Omega$, we denote by $V_{\xi}$ the maximal vector subspace of $\mathbf{R}^{n}$ such that $\xi+V_{\xi} \subset \Omega$, then the vector subspaces $V_{\xi}, \xi \in \Omega$ coincide to each other, which we denote by $V(D)$, and its dimension is equal to $l$. As a consequence, $l$ is independent of the choice of $f$ satisfying (2.1). Therefore the integer $l$ is an invariant associated with $D$, which we denote by $l(D)$.

Here are some observations about $l(D)$. Let $D$ be a pseudoconvex Reinhardt domain in $\left(\mathbf{C}^{*}\right)^{n}$ and write $\Omega=\operatorname{ord}(D)$. When $l(D)=0$, the domain $D$ is algebraically equivalent to a bounded Reinhardt domain in $\left(\mathbf{C}^{*}\right)^{n}$ (cf. [1]). On the other hand, when $l(D)>0$, write an affine transformation $f$ of $\mathbf{R}^{n}$ satisfying (2.1) as $f(\xi)=L \xi+b$ for $\xi \in \mathbf{R}^{n}$, where $L \in G L(n, \mathbf{R})$ and $b \in \mathbf{R}^{n}$. If we define an affine transformation $F$ of $\mathbf{C}^{n}$ by $F(\zeta)=L \zeta+b$ for $\zeta \in \mathbf{C}^{n}$, then we have

$$
F\left(T_{\Omega}\right)=T_{f(\Omega)}=T_{\Xi^{(1)} \times \mathbf{R}^{l(D)}}=T_{\Xi^{(1)}} \times T_{\mathbf{R}^{l(D)}}=T_{\Xi^{(1)}} \times \mathbf{C}^{l(D)},
$$

and hence $T_{\Omega}$ is holomorphically equivalent to $T_{\Xi^{(1)}} \times \mathbf{C}^{l(D)}$. Note that $l(D)=n$ if and only if $D=\left(\mathbf{C}^{*}\right)^{n}$.

The following lemma is easily proved by using the notion of Liouville foliation introduced in Shimizu [4] (cf. [5]).

Lemma 2.1. Let $E \times \mathbf{C}^{l}$ and $E^{\prime} \times \mathbf{C}^{l^{\prime}}$ be two domains in $\mathbf{C}^{n}$, where $E$ and $E^{\prime}$ are domains in $\mathbf{C}^{n-l}$ and $\mathbf{C}^{n-l^{\prime}}$, respectively, that are holomorphically equivalent to bounded domains. Suppose that there is a biholomorphic mapping $\Phi$ of $E \times \mathbf{C}^{l}$ onto $E^{\prime} \times \mathbf{C}^{l^{\prime}}$. Then $l$ and $l^{\prime}$ coincide. Moreover, if each point $w \in \mathbf{C}^{n}=$ $\mathbf{C}^{n-l} \times \mathbf{C}^{l}$ is written as

$$
w=\left(\begin{array}{c}
w^{(1)} \\
w^{(2)}
\end{array}\right), \quad w^{(1)} \in \mathbf{C}^{n-l}, w^{(2)} \in \mathbf{C}^{l},
$$

then $\Phi$ has the form

$$
\Phi: E \times \mathbf{C}^{l} \ni w=\left(\begin{array}{c}
w^{(1)} \\
w^{(2)}
\end{array}\right) \mapsto\left(\begin{array}{c}
\Phi^{(1)}\left(w^{(1)}\right) \\
\Phi^{(2)}(w)
\end{array}\right) \in E^{\prime} \times \mathbf{C}^{l^{\prime}},
$$

where $\Phi^{(1)}: E \ni w^{(1)} \mapsto \Phi^{(1)}\left(w^{(1)}\right) \in E^{\prime}$ gives a biholomorphic mapping of $E$ onto $E^{\prime}$.

As an immediate consequence of this lemma, we see that $l(D)$ is a biholomorphic invariant:

Corollary 2.1. If two pseudoconvex Reinhardt domains $D$ and $D^{\prime}$ in $\left(\mathbf{C}^{*}\right)^{n}$ are holomorphically equivalent, then $l(D)$ and $l\left(D^{\prime}\right)$ coincide. 
We denote by $G L(n, \mathbf{Z}) \ltimes \mathbf{C}^{n}$ the group of all complex affine transformations of $\mathbf{C}^{n}$ whose linear parts belong to $G L(n, \mathbf{Z})$. We discuss the relation between Aut $_{\mathrm{alg}}\left(\left(\mathbf{C}^{*}\right)^{n}\right)$ and $G L(n, \mathbf{Z}) \ltimes \mathbf{C}^{n}$. Let $\Phi$ be any element of $G L(n, \mathbf{Z}) \ltimes \mathbf{C}^{n}$ and write $\Phi(\zeta)=A \zeta+\beta$ for $\zeta \in \mathbf{C}^{n}$, where $A=\left(a_{i j}\right) \in G L(n, \mathbf{Z})$ and $\beta=\left(\beta_{i}\right) \in \mathbf{C}^{n}$. Then we can define an element $\varphi$ of $\operatorname{Aut}_{\mathrm{alg}}\left(\left(\mathbf{C}^{*}\right)^{n}\right)$ by

$$
\begin{gathered}
\varphi:\left(\mathbf{C}^{*}\right)^{n} \ni{ }^{t}\left(z_{1}, \ldots, z_{n}\right) \mapsto{ }^{t}\left(w_{1}, \ldots, w_{n}\right) \in\left(\mathbf{C}^{*}\right)^{n}, \\
w_{i}=e^{-2 \pi \beta_{i}} z_{1}^{a_{i 1}} \cdots z_{n}^{a_{i n}}, \quad i=1, \ldots, n .
\end{gathered}
$$

The mapping $\rho: G L(n, \mathbf{Z}) \ltimes \mathbf{C}^{n} \rightarrow \operatorname{Aut}_{\mathrm{alg}}\left(\left(\mathbf{C}^{*}\right)^{n}\right)$ sending $\Phi$ to $\varphi$ is a group homomorphism of $G L(n, \mathbf{Z}) \ltimes \mathbf{C}^{n}$ onto $\operatorname{Aut}_{\mathrm{alg}}\left(\left(\mathbf{C}^{*}\right)^{n}\right)$. Note that the kernel of $\rho$ is given by $\left\{\sigma_{\eta} \mid \eta \in \mathbf{Z}^{n}\right\} \subset \operatorname{Aut}\left(\mathbf{C}^{n}\right)$, and that $\Phi, \rho(\Phi)$, and the covering projection $\varpi: \mathbf{C}^{n} \rightarrow\left(\mathbf{C}^{*}\right)^{n}$ commute in the following sense:

$$
\varpi \circ \Phi=\rho(\Phi) \circ \varpi \text { for every } \Phi \in G L(n, \mathbf{Z}) \ltimes \mathbf{C}^{n} .
$$

If $D$ and $D^{\prime}$ are Reinhardt domains in $\left(\mathbf{C}^{*}\right)^{n}$ with the covering tube domains $T_{\Omega}$ and $T_{\Omega^{\prime}}$, respectively, and if $\Phi$ is an element of $G L(n, \mathbf{Z}) \ltimes \mathbf{C}^{n}$, then, by (2.2), we have $\rho(\Phi)(D)=D^{\prime}$ precisely when $\Phi\left(T_{\Omega}\right)=T_{\Omega^{\prime}}$. As a consequence of this, we see that if there exists an element $\Phi$ of $G L(n, \mathbf{Z}) \ltimes \mathbf{C}^{n}$ such that $\Phi\left(T_{\Omega}\right)=T_{\Omega^{\prime}}$, then $D$ and $D^{\prime}$ are algebraically equivalent.

To discuss the correspondence of biholomorphic mappings between Reinhardt domains with biholomorphic mappings between tube domains, let $D$ and $D^{\prime}$ be two Reinhardt domains in $\left(\mathbf{C}^{*}\right)^{n}$ and let $T_{\Omega}$ and $T_{\Omega^{\prime}}$ denote the covering tube domains of $D$ and $D^{\prime}$, respectively. Suppose $\Phi: T_{\Omega} \rightarrow T_{\Omega^{\prime}}$ is a biholomorphic mapping between $T_{\Omega}$ and $T_{\Omega^{\prime}}$ and satisfies the condition that, for some $A \in$ $G L(n, \mathbf{Z})$, we have

$$
\Phi(\zeta+\sqrt{-1} m)=\Phi(\zeta)+\sqrt{-1} A m \text { for every } \zeta \in T_{\Omega} \text { and every } m \in \mathbf{Z}^{n}
$$

Then, since the covering transformation groups for $\varpi: T_{\Omega} \rightarrow D$ and $\varpi: T_{\Omega^{\prime}} \rightarrow$ $D^{\prime}$ are given by $\left\{\sigma_{\eta} \mid \eta \in \mathbf{Z}^{n}\right\} \subset \operatorname{Aut}\left(T_{\Omega}\right)$ and $\left\{\sigma_{\eta} \mid \eta \in \mathbf{Z}^{n}\right\} \subset \operatorname{Aut}\left(T_{\Omega^{\prime}}\right)$, respectively, it follows that there exists a biholomorphic mapping $\varphi: D \rightarrow D^{\prime}$ between $D$ and $D^{\prime}$ such that $\varpi \circ \Phi=\varphi \circ \varpi$. Conversely, when $D$ and $D^{\prime}$ are pseudoconvex, every biholomorphic mapping $\varphi: D \rightarrow D^{\prime}$ between $D$ and $D^{\prime}$ has a lifting $\Phi: T_{\Omega} \rightarrow T_{\Omega^{\prime}}$, or a biholomorphic mapping $\Phi$ of $T_{\Omega}$ onto $T_{\Omega^{\prime}}$ such that $\varpi \circ \Phi=$ $\varphi \circ \varpi$ and satisfying (2.3) for some $A \in G L(n, \mathbf{Z})$, because $\varpi: T_{\Omega} \rightarrow D$ and $\varpi: T_{\Omega^{\prime}} \rightarrow D^{\prime}$ are the universal coverings of $D$ and $D^{\prime}$, respectively. As a consequence of these observations and [2, Section 6, Corollary to Theorem 2 ], we have the following proposition, which gives a useful tool in our investigation.

Proposition 2.2. Let $\Phi: T_{\Omega} \rightarrow T_{\Omega^{\prime}}$ be a biholomorphic mapping between two tube domains $T_{\Omega}$ and $T_{\Omega^{\prime}}$ in $\mathbf{C}^{n}$ whose bases $\Omega$ and $\Omega^{\prime}$ have the convex hulls containing no complete straight lines. Suppose that there exist elements $A$ and $B$ 
of $G L(n, \mathbf{R})$ such that

$$
\Phi(\zeta+\sqrt{-1} A m)=\Phi(\zeta)+\sqrt{-1} B m \quad \text { for every } \zeta \in T_{\Omega} \text { and every } m \in \mathbf{Z}^{n} .
$$

Then $\Phi$ is an affine transformation of $\mathbf{C}^{n}$ whose linear part belongs to $G L(n, \mathbf{R})$.

Proof. The proof of this proposition is given in Shimizu [5]. Although there are overlaps with that, we carry out the proof in detail for the sake of completeness and self-containedness.

We define automorphisms $F_{A}$ and $F_{B}$ of $\mathbf{C}^{n}$ given as linear transformations by

$$
F_{A}(w)=A w \quad \text { for } w \in \mathbf{C}^{n} \quad \text { and } \quad F_{B}(\omega)=B \omega \quad \text { for } \omega \in \mathbf{C}^{n} .
$$

Then the domains $F_{A}^{-1}\left(T_{\Omega}\right)$ and $F_{B}^{-1}\left(T_{\Omega^{\prime}}\right)$ in $\mathbf{C}^{n}$ are tube domains. Indeed, writing $\Xi=A^{-1} \Omega$ and $\Xi^{\prime}=B^{-1} \Omega^{\prime}$, we have $F_{A}^{-1}\left(T_{\Omega}\right)=T_{\Xi}$ and $F_{B}^{-1}\left(T_{\Omega^{\prime}}\right)=T_{\Xi^{\prime}}$. Note that, since $\Omega$ and $\Omega^{\prime}$ have the convex hulls containing no complete straight lines, $\Xi$ and $\Xi^{\prime}$ also have the convex hulls containing no complete straight lines. We set $E=\varpi\left(T_{\Xi}\right)$ and $E^{\prime}=\varpi\left(T_{\Xi^{\prime}}\right)$. By the definition of $\varpi$, the domains $E$ and $E^{\prime}$ are Reinhardt domains in $\left(\mathbf{C}^{*}\right)^{n}$, and $T_{\Xi}$ and $T_{\Xi^{\prime}}$ are the covering tube domains of $E$ and $E^{\prime}$, respectively. An application of [2, Section 6, Corollary to Theorem 2] to $E$ and $E^{\prime}$ yields that every biholomorphic mapping of $E$ onto $E^{\prime}$ is induced by an algebraic automorphism of $\left(\mathbf{C}^{*}\right)^{n}$.

Now consider a biholomorphic mapping $\Psi: T_{\Xi} \rightarrow T_{\Xi^{\prime}}$ between $T_{\Xi}$ and $T_{\Xi^{\prime}}$ given by $\Psi=F_{B}^{-1} \circ \Phi \circ F_{A}$. Then we see from (2.4) that $\Psi$ satisfies the condition that

$$
\Psi(w+\sqrt{-1} m)=\Psi(w)+\sqrt{-1} m \text { for every } w \in T_{\Xi} \text { and every } m \in \mathbf{Z}^{n} .
$$

Therefore, as observed above, there exists a biholomorphic mapping $\psi: E \rightarrow E^{\prime}$ between $E$ and $E^{\prime}$ such that $\varpi \circ \Psi=\psi \circ \varpi$. By the result of the preceding paragraph, $\psi$ is induced by an algebraic automorphism of $\left(\mathbf{C}^{*}\right)^{n}$. This implies that we can find an element $\Psi_{0}$ of $G L(n, \mathbf{Z}) \ltimes \mathbf{C}^{n}$ such that $\Psi_{0}\left(T_{\Xi}\right)=T_{\Xi^{\prime}}$ and $\varpi \circ \Psi_{0}=\psi \circ \varpi$ on $T_{\Xi}$. Note that both $\Psi: T_{\Xi} \rightarrow T_{\Xi^{\prime}}$ and $\Psi_{0}: T_{\Xi} \rightarrow T_{\Xi^{\prime}}$ are liftings of $\psi$. Replacing, if necessary, $\Psi_{0}$ by $\sigma_{\eta} \circ \Psi_{0}$ for some $\eta \in \mathbf{Z}^{n}$, we may assume that $\Psi\left(w_{0}\right)=\Psi_{0}\left(w_{0}\right)$ for a point $w_{0}$ of $T_{\Xi}$. Then we see by the uniqueness of lifting that $\Psi=\Psi_{0}$, or $\Phi=F_{B} \circ \Psi_{0} \circ F_{A}^{-1}$. Since $F_{B} \circ \Psi_{0} \circ F_{A}^{-1}$ is an affine transformation of $\mathbf{C}^{n}$, this completes the proof of the proposition.

To apply the above proposition to our proof of Theorem 1.2, we need a lemma.

LeMma 2.2. Let $\Phi: T_{\Omega} \rightarrow T_{\Omega^{\prime}}$ be a biholomorphic mapping between two tube domains $T_{\Omega}$ and $T_{\Omega^{\prime}}$ in $\mathbf{C}^{n}$. Suppose that there exists a real $n \times n$ matrix $A$ such that the condition (2.3) holds. Then the matrix $A$ is non-singular. 
Proof. We denote by $\operatorname{Ker} A$ the kernel of the linear transformation of $\mathbf{R}^{n}$ determined by $A$. Suppose that $\operatorname{Ker} A \neq\{0\}$ and we shall derive a contradiction.

Consider first the case where $\operatorname{Ker} A \cap \mathbf{Z}^{n} \neq\{0\}$. Then, taking a non-zero element $m$ of $\operatorname{Ker} A \cap \mathbf{Z}^{n}$, we see from (2.3) that

$$
\Phi(\zeta+\sqrt{-1} m)=\Phi(\zeta)+\sqrt{-1} A m=\Phi(\zeta) \text { for } \zeta \in T_{\Omega},
$$

which contradicts the assumption that $\Phi$ is injective.

Consider next the case where $\operatorname{Ker} A \cap \mathbf{Z}^{n}=\{0\}$. Let $H$ be the closure $\overline{A \mathbf{Z}^{n}}$ of $A \mathbf{Z}^{n}$ in $\mathbf{R}^{n}$. Then $H$ is a closed subgroup of the vector group $A \mathbf{R}^{n}$. Consequently, $H$ is a Lie subgroup of the abelian Lie group $A \mathbf{R}^{n}$. Note that the identity component of $H$ is a linear subspace of $A \mathbf{R}^{n}$.

When $\operatorname{dim} H=0$, the group $H$ is a lattice with

$$
\text { rank } H \leq \operatorname{dim} A \mathbf{R}^{n}<n \text {. }
$$

On the other hand, $H$ contains the subgroup $A \mathbf{Z}^{n}$, which is isomorphic to the lattice $\mathbf{Z}^{n}$, because $\operatorname{Ker} A \cap \mathbf{Z}^{n}=\{0\}$ by the assumption. Therefore we have rank $H \geq n$. This contradicts (2.5).

Suppose that $\operatorname{dim} H>0$. Fix a point $\zeta_{0}$ of $T_{\Omega}$ and take a neighborhood $W$ of $\zeta_{0}$ such that $W \cap\left(\zeta_{0}+\sqrt{-1} \mathbf{Z}^{n}\right)=\left\{\zeta_{0}\right\}$. Since $\Phi: T_{\Omega} \rightarrow T_{\Omega^{\prime}}$ is a biholomorphic mapping, $\Phi(W)$ is a neighborhood of $\Phi\left(\zeta_{0}\right)$ and we have

$$
\Phi(W) \cap \Phi\left(\zeta_{0}+\sqrt{-1} \mathbf{Z}^{n}\right)=\left\{\Phi\left(\zeta_{0}\right)\right\} .
$$

By $H=\overline{A \mathbf{Z}^{n}}$ and $\operatorname{dim} H>0$, there exists a non-zero element of $A \mathbf{Z}^{n}$ arbitrarily close to 0 . Therefore, since $\Phi\left(\zeta_{0}+\sqrt{-1} m\right)=\Phi\left(\zeta_{0}\right)+\sqrt{-1} A m$ by (2.3), there exists an element of $\Phi\left(\zeta_{0}+\sqrt{-1} \mathbf{Z}^{n}\right)$ arbitrarily close to $\Phi\left(\zeta_{0}\right)$ which does not coincide with $\Phi\left(\zeta_{0}\right)$ itself. This contradicts (2.6), and the lemma is proved.

\section{Proof of Theorem $\mathbf{1 . 2}$}

Let $D$ be a pseudoconvex homogeneous Reinhardt domain in $\left(\mathbf{C}^{*}\right)^{n}$. Then, $D$ coinsides with $\left(\mathbf{C}^{*}\right)^{n}$ if and only if $l(D)=n$. Therefore, suppose $l(D)<n$ and we shall derive a contradiction.

If $l(D)=0$, then $D$ is algebraically equivalent to a bounded Reinhardt domain. By Theorem 1.1, every homogeneous bounded Reinhardt domain is algebraically equivalent to the direct product $D^{\prime}:=B_{n_{1}} \times \cdots \times B_{n_{k}}$ of balls, which contains the origin. Hence there exists a holomorphic isomorphism of $D$ onto $D^{\prime}$ induced by an algebraic automorphism $\left(z_{i}\right) \mapsto\left(w_{i}\right)$ of $\left(\mathbf{C}^{*}\right)^{n}$ of the form $w_{i}=\alpha_{i} z_{\tau(i)}, i=1, \ldots, n$, where $\tau$ is a permutation of $\{1,2, \ldots, n\}$ and $\left(\alpha_{1}, \ldots, \alpha_{n}\right) \in\left(\mathbf{C}^{*}\right)^{n}$. Consequently, $D$ contains the origin and this contradicts the assumption that $D \subset\left(\mathbf{C}^{*}\right)^{n}$.

Now we suppose $0<l(D)<n$ and set $k=n-l(D)$. We divide the proof into four steps. 
STEP 1. Let $T_{\Omega}$ be the covering tube domain of $D$ and let $\Gamma:=\left\{\sigma_{\eta} \mid \eta \in \mathbf{Z}^{n}\right\}$ be its covering transformarion group. We denote by $\tilde{\varphi} \in \operatorname{Aut}\left(T_{\Omega}\right)$ a lifting of $\varphi \in \operatorname{Aut}(D)$. The set $G$ of all liftings $\tilde{\varphi}$ forms a subgroup of $\operatorname{Aut}\left(T_{\Omega}\right)$ and is given as the normalizer of $\Gamma$ in $\operatorname{Aut}\left(T_{\Omega}\right)$. Since the covering transformation group $\Gamma$ is isomorphic to the additive group $\mathbf{Z}^{n}$, for every $\tilde{\varphi} \in G$ there exists a unique $A \in G L(n, \mathbf{Z})$ such that

$$
\tilde{\varphi} \circ \sigma_{\eta} \circ \tilde{\varphi}^{-1}=\sigma_{A \eta} .
$$

From this, we have a linear representation $\rho$ of $G$ on $G L(n, \mathbf{Z})$ such that $G \ni \tilde{\varphi} \mapsto A \in G L(n, \mathbf{Z})$. By the assumption, $\operatorname{Aut}(D)$ acts on $D$ transitively. Therefore $G$ acts on $T_{\Omega}$ transitively.

Next, we shall see what influences a permutation of coordinates has on the linear representation $\rho$ of $G$. We consider a linear transformation of $\mathbf{C}^{n}$ represented by a matrix $P_{\tau}:=\left(\delta_{\tau(i) j}\right) \in G L(n, \mathbf{Z})$, where $\tau$ is a permutation of $\{1,2, \ldots, n\}$. Then, the universal covering $\varpi: T_{\Omega} \rightarrow D$ is replaced by the covering $P_{\tau} \circ \varpi \circ P_{\tau}^{-1}: T_{P_{\tau}(\Omega)} \rightarrow P_{\tau}(D)$, and a lifting of $P_{\tau} \circ \varphi \circ P_{\tau}^{-1} \in \operatorname{Aut}\left(P_{\tau}(D)\right)$ is given by $P_{\tau} \circ \tilde{\varphi} \circ P_{\tau}^{-1} \in \operatorname{Aut}\left(T_{P_{\tau}(\Omega)}\right)$. The equation (3.1) means that

$$
\tilde{\varphi}(\zeta+\sqrt{-1} m)=\tilde{\varphi}(\zeta)+\sqrt{-1} A m
$$

for every $\zeta \in T_{\Omega}$ and for every $m \in \mathbf{Z}^{n}$. Hence we have

$$
\begin{aligned}
P_{\tau} \circ \tilde{\varphi} \circ P_{\tau}^{-1}(\zeta+\sqrt{-1} m) & =P_{\tau} \circ \tilde{\varphi}\left(P_{\tau}^{-1}(\zeta)+\sqrt{-1} P_{\tau}^{-1}(m)\right) \\
& =P_{\tau}\left(\tilde{\varphi}\left(P_{\tau}^{-1}(\zeta)\right)+\sqrt{-1} A P_{\tau}^{-1}(m)\right) \\
& =P_{\tau} \circ \tilde{\varphi} \circ P_{\tau}^{-1}(\zeta)+\sqrt{-1} P_{\tau} A P_{\tau}^{-1} m
\end{aligned}
$$

for every $\zeta \in T_{P_{\tau}(\Omega)}$ and for every $m \in \mathbf{Z}^{n}$. Consequently the linear representation $\rho: G \rightarrow G L(n, \mathbf{Z})$ is just replaced by $\rho^{\prime}: P_{\tau} G P_{\tau}^{-1} \ni P_{\tau} \circ \tilde{\varphi} \circ P_{\tau}^{-1} \mapsto P_{\tau} A P_{\tau}^{-1} \in$ $G L(n, \mathbf{Z})$.

STEP 2. We would like to represent the domain $\Omega=\operatorname{ord}(D)$ in $\mathbf{R}^{n}$ as simple as possible. By means of a linear transformation $L$ on $\mathbf{R}^{n}$ induced by a suitable permutation of coordinates, we make $V(D)$ parallel to some coordinate axes.

For simplicity, write $l:=l(D)$. Since $\operatorname{dim} V(D)=l$, there exists a basis $\left\{v_{1}, \ldots, v_{l}\right\}$ of $V(D)$ over $\mathbf{R}$. We write $V:=\left(v_{1}, \ldots, v_{l}\right)$, which is an $n \times l$ matrix consisting of column vectors $v_{1}, \ldots, v_{l}$. As rank $V=l$, doing a suitable permutation of coordinates, we have

$$
V=\left(\begin{array}{l}
V^{(1)} \\
V^{(2)}
\end{array}\right),
$$

where $V^{(1)}$ is a $k \times l$ matrix and $V^{(2)}$ is a non-singular $l \times l$ matrix. Moreover, by means of elementary transformations to column vectors of $V$ if necessary, we have $V^{(2)}=I_{l}$ and

$$
V=\left(\begin{array}{c}
V^{(1)} \\
I_{l}
\end{array}\right) .
$$


We define a matrix $L$ by

$$
L:=\left(\begin{array}{cc}
I_{k} & -V^{(1)} \\
O & I_{l}
\end{array}\right) \in G L(n, \mathbf{R})
$$

Then we see

$$
L V=\left(\begin{array}{cc}
I_{k} & -V^{(1)} \\
O & I_{l}
\end{array}\right)\left(\begin{array}{c}
V^{(1)} \\
I_{l}
\end{array}\right)=\left(\begin{array}{c}
O \\
I_{l}
\end{array}\right) .
$$

Writing $\Xi:=L \Omega$, we have

$$
\Xi=\Xi^{(1)} \times \mathbf{R}^{l}
$$

where $\Xi^{(1)}$ is a convex domain in $\mathbf{R}^{k}$ containing no complete straight lines. If we consider $L$ as a linear transformation of $\mathbf{C}^{n}$, then $L\left(T_{\Omega}\right)=T_{L(\Omega)}=T_{\Xi}=$ $T_{\Xi^{(1)}} \times \mathbf{C}^{l}$, and $\Phi:=L \circ \tilde{\varphi} \circ L^{-1} \in \operatorname{Aut}\left(T_{\Xi}\right)$ satisfies

$$
\Phi(w+\sqrt{-1} L m)=\Phi(w)+\sqrt{-1} L A m
$$

for every $w \in T_{\Xi}$ and for every $m \in \mathbf{Z}^{n}$. Indeed, by (3.2),

$$
\begin{aligned}
\Phi(w+\sqrt{-1} L m) & =L \circ \tilde{\varphi} \circ L^{-1}(w+\sqrt{-1} L m) \\
& =L \circ \tilde{\varphi}\left(L^{-1} w+\sqrt{-1} m\right) \\
& =L\left(\tilde{\varphi}\left(L^{-1} w\right)+\sqrt{-1} A m\right) \\
& =\Phi(w)+\sqrt{-1} L A m .
\end{aligned}
$$

Note that $L G L^{-1}$ acts on $T_{\Xi}$ transitively.

STEP 3. We consider a holomorphic autmorphism:

$$
\Phi: T_{\Xi^{(1)}} \times \mathbf{C}^{l} \ni w=\left(\begin{array}{c}
w^{(1)} \\
w^{(2)}
\end{array}\right) \mapsto\left(\begin{array}{l}
\Phi^{(1)}(w) \\
\Phi^{(2)}(w)
\end{array}\right) \in T_{\Xi^{(1)}} \times \mathbf{C}^{l},
$$

where $w^{(1)}, \Phi^{(1)}(w) \in T_{\Xi^{(1)}}$ and $w^{(2)}, \Phi^{(2)}(w) \in \mathbf{C}^{l}$. Since $\Xi^{(1)}$ is a convex domain in $\mathbf{R}^{k}$ containing no complete straight lines, $T_{\Xi^{(1)}}$ is holomorphically equivalent to a bounded domain in $\mathbf{C}^{k}$. Hence, Lemma 2.1 implies that the first component $\Phi^{(1)}$ depends only on $w^{(1)}$ and $\Phi^{(1)}: T_{\Xi^{(1)}} \ni w^{(1)} \mapsto \Phi^{(1)}\left(w^{(1)}\right) \in T_{\Xi^{(1)}}$ is a holomorphic automorphism.

We shall see a more precise form of $\Phi^{(1)}$. For every $A=\rho(\tilde{\varphi})$ with $\tilde{\varphi} \in G$, set

$$
L A=:\left(\begin{array}{ll}
A^{(1)} & A^{(2)} \\
A^{(3)} & A^{(4)}
\end{array}\right),
$$

where $A^{(1)}$ is a $k \times k$ matrix, and $A^{(2)}, A^{(3)}, A^{(4)}$ are $k \times l, l \times k, l \times l$ matrices respectively. Note that the matrix $L$ defined by (3.3) is independent of $A$. We 
see that $A^{(1)}$ is an element of $G L(k, \mathbf{R})$ later. Set

$$
L^{(1)}:=\left(I_{k},-V^{(1)}\right), \quad(L A)^{(1)}:=\left(A^{(1)}, A^{(2)}\right) .
$$

Then, by (3.5), $\Phi^{(1)}$ satisfies

$$
\Phi^{(1)}\left(w^{(1)}+\sqrt{-1} L^{(1)} m\right)=\Phi^{(1)}\left(w^{(1)}\right)+\sqrt{-1}(L A)^{(1)} m
$$

for every $w^{(1)} \in T_{\Xi^{(1)}}$ and for every $m \in \mathbf{Z}^{n}$. In particular, putting

$$
m={ }^{t}\left(m_{1}, \ldots, m_{k}, 0, \ldots, 0\right),
$$

and writing $m^{(1)}:={ }^{t}\left(m_{1}, \ldots, m_{k}\right)$, by (3.7) we have

$$
L^{(1)} m=m^{(1)}, \quad(L A)^{(1)} m=A^{(1)} m^{(1)} .
$$

Hence, (3.8) implies that

$$
\Phi^{(1)}\left(w^{(1)}+\sqrt{-1} m^{(1)}\right)=\Phi^{(1)}\left(w^{(1)}\right)+\sqrt{-1} A^{(1)} m^{(1)}
$$

for every $w^{(1)} \in T_{\Xi^{(1)}}$ and for every $m^{(1)} \in \mathbf{Z}^{k}$, and, by Lemma 2.2, the matrix $A^{(1)}$ is non-singular. By Proposition 2.2, we see $\Phi^{(1)} \in G L(k, \mathbf{R}) \ltimes \mathbf{C}^{k}$, that is, there exist $B^{(1)} \in G L(k, \mathbf{R})$ and $\beta^{(1)} \in \mathbf{C}^{k}$ such that

$$
\Phi^{(1)}\left(w^{(1)}\right)=B^{(1)} w^{(1)}+\beta^{(1)} .
$$

Substituting (3.10) into (3.9) yields $B^{(1)} m^{(1)}=A^{(1)} m^{(1)}$ for every $m^{(1)} \in \mathbf{Z}^{k}$. Consequently, we see that $B^{(1)}=A^{(1)}$, or

$$
\Phi^{(1)}\left(w^{(1)}\right)=A^{(1)} w^{(1)}+\beta^{(1)} \text { for every } w^{(1)} \in T_{\Xi^{(1)}} .
$$

Note that $G^{(1)}:=\left\{\Phi^{(1)} \mid \Phi \in L G L^{-1}\right\}$ acts on $T_{\Xi^{(1)}}$ transitively.

In (3.11), we can decompose $\Phi^{(1)}$ into real and imaginary components, since $A^{(1)} \in G L(k, \mathbf{R})$. Namely, write

$$
w^{(1)}=: \xi^{(1)}+\sqrt{-1} \eta^{(1)}, \quad \beta^{(1)}=: a^{(1)}+\sqrt{-1} b^{(1)},
$$

where $\xi^{(1)}, \eta^{(1)}, a^{(1)}, b^{(1)}$ are elements of $\mathbf{R}^{k}$. Then, we have

$$
\Phi^{(1)}\left(w^{(1)}\right)=A^{(1)} \xi^{(1)}+a^{(1)}+\sqrt{-1}\left(A^{(1)} \eta^{(1)}+b^{(1)}\right)
$$

and the real component

$$
\operatorname{Re} \Phi^{(1)}\left(\xi^{(1)}\right):=A^{(1)} \xi^{(1)}+a^{(1)}
$$

gives an affine automorphism of the domain $\Xi^{(1)}$. Then, $H^{(1)}:=\left\{\operatorname{Re} \Phi^{(1)} \mid\right.$ $\left.\Phi^{(1)} \in G^{(1)}\right\}$ acts on $\Xi^{(1)}$ transitively.

SteP 4. We see that in (3.12), the translational part $a^{(1)}$ is uniquely determined by the linear part $A^{(1)}$ :

Lemma 3.1. If $A^{(1)} \xi^{(1)}+a^{(1)}$ and $A^{(1)} \xi^{(1)}+b^{(1)}$ are elements of $H^{(1)}$, then $a^{(1)}=b^{(1)}$. 
Proof. By the assumptions, we have

$$
A^{(1)}\left(\Xi^{(1)}\right)+a^{(1)}=\Xi^{(1)}, \quad A^{(1)}\left(\Xi^{(1)}\right)+b^{(1)}=\Xi^{(1)} .
$$

Since $\Xi^{(1)}-a^{(1)}=A^{(1)}\left(\Xi^{(1)}\right)$, it follows that

$$
\Xi^{(1)}+\left(b^{(1)}-a^{(1)}\right)=\left(\Xi^{(1)}-a^{(1)}\right)+b^{(1)}=A^{(1)}\left(\Xi^{(1)}\right)+b^{(1)}=\Xi^{(1)} .
$$

Since $\Xi^{(1)}$ is a convex domain containning no complete straight lines, this can only happen when $b^{(1)}-a^{(1)}=0$.

Note that in (3.6), $A^{(1)}$ is the $k \times k$ principal matrix of $L A$, where $L \in G L(n, \mathbf{R})$ is the fixed matrix determined by the domain $D$ and $A \in \rho(G) \subset$ $G L(n, \mathbf{Z})$. By Lemma 3.1, we have a surjection of $\rho(G)$ onto $H^{(1)}$. Since $\rho(G)$ is at most countable, so is $H^{(1)}$. This contradicts the fact that $H^{(1)}$ acts on $\Xi^{(1)}$ transitively, and the proof of Theorem 1.2 completely finished.

\title{
REFERENCES
}

[1] M. JARnicki AND P. Pflug, First steps in several complex variables: Reinhardt domains, European Mathematical Society, 2008.

[2] S. Shimizu, Automorphisms and equivalence of bounded Reinhardt domains not containing the origin, Tohoku Math. J. 40 (1988), 119-152.

[ 3 ] S. Shimizu, Automorphisms of bounded Reinhardt domains, Japan. J. Math. 15 (1989), $385-414$.

[4] S. Shimizu, Holomorphic equivalence problem for a certain class of unbounded Reinhardt domains in $\mathbf{C}^{2}$, Osaka J. Math. 28 (1991), 609-621.

[5] S. SHImizu, Holomorphic equivalence problem for Reinhardt domains and the conjugacy of torus actions, preprint.

[6] T. Sunada, Holomorphic equivalence problem for bounded Reinhardt domains, Math. Ann. 235 (1978), 111-128.

\author{
Satoru Shimizu \\ Mathematical INSTITUTE \\ TOHOKU UNIVERSITY \\ SENDAI 980-8578 \\ JAPAN \\ E-mail: shimizu@math.tohoku.ac.jp \\ Kouichi Kimura \\ Mathematical Institute \\ TOHOKU UNIVERSITY \\ SENDAI 980-8578 \\ JAPAN \\ E-mail: sa9m12@math.tohoku.ac.jp
}

